\title{
Cardiac physiology in post myocardial infarction patients: the effect of cardiac rehabilitation programs-a systematic review and update meta-analysis
}

\author{
Irene Kirolos ${ }^{1}$, Danny Yakoub ${ }^{2}$, Fiorella Pendola ${ }^{3}$, Omar Picado ${ }^{4}$, Aghapy Kirolos ${ }^{1}$, Yehoshua C. Levine ${ }^{1}$, \\ Sunil Jha ${ }^{1}$, Rajesh Kabra ${ }^{1}$, Brandon Cave ${ }^{1}$, Rami N. Khouzam ${ }^{1}$ \\ ${ }^{1}$ Division of Cardiovascular Diseases, Department of Medicine, University of Tennessee Health Science Center, Cardiovascular Institute, Methodist \\ Le Bonheur Healthcare System, Memphis, TN, USA; ${ }^{2}$ Division of Surgical Oncology, Department of Surgery, University of Tennessee Health \\ Science Center, Memphis, TN, USA; ${ }^{3}$ Department of Internal Medicine, Blake Medical Center, Brandon, FL, USA; ${ }^{4}$ Department of Surgery, \\ Jackson Memorial Hospital, University of Miami - Miller School of Medicine, Miami, FL, USA \\ Contributions: (I) Conception and design: I Kirolos, D Yakoub, F Pendola; (II) Administrative support: R Khouzam, R Kabra; (III) Provision of \\ study materials: Y Levine, S Jha, R Kabra, R Khouzam; (IV) Collection and assembly of data: I Kirolos, F Pendola, A Kirolos; (V) Data analysis and \\ interpretation: I Kirolos, D Yakoub, O Picado; (VI) Manuscript writing: All authors; (VII) Final approval of manuscript: All authors. \\ Correspondence to: Irene Kirolos, MD, MPH. Division of Cardiology, University of Tennessee Health Science Center, Coleman Building, 956 Court \\ Avenue, Memphis, TN 38163, USA. Email: ikirolos@uthsc.edu.
}

\begin{abstract}
Cardiac rehabilitation program (CRP) is a recognized non-pharmacological modality to decrease mortality after acute myocardial infarction (AMI) events. We aimed to evaluate the effect of CRP on the cardiac physiology in patients post myocardial infarction (MI). Online database search of PubMed, MEDLINE, EMBASE, SCOPUS, COCHRANE, and GOOGLE SCHOLAR were performed (1988-Mar 2016); key bibliographies were reviewed. Studies comparing post MI patients who were enrolled in a CRP to those who were not, were included. Standardized mean difference (SMD) with the corresponding $95 \%$ confidence intervals (CI) by random and fixed effects models of pooled data were calculated. Study quality was assessed using CONSORT criteria. Outcomes of interest measured included resting and maximum heart rate (HR), peak $\mathrm{VO}_{2}$, ejection fraction (EF\%), wall motion score index (WMSI), left ventricular end diastolic volume (LVEDV) in cardiac rehabilitation patients versus control. Search strategy yielded 147 studies, 23 studies fulfilled the selection criteria, 19 of which were RCTs. These included a total of 1,683 patients; 827 were enrolled in a CRP while 855 did not receive the intervention. Median age was 58 years. There was no significant difference between the two groups in terms of age, comorbidities, severity of CAD, baseline EF or HR. Meta-analysis of data included demonstrated that CRP patients had lower post-intervention resting HR than non-CRP patients (SMD: $-0.59 ; 95 \%$ CI: -0.73 to -0.46 , fixed effect model $\mathrm{P}<0.05)$. EF\% was significantly improved after CRP compared to control (SMD: 0.21; 95\% CI: 0.02 to $0.40, \mathrm{P}=0.03$ ). Peak $\mathrm{VO}_{2}$ was significantly improved by CRP (SMD: $1.00 ; 95 \% \mathrm{CI}$ : 0.56 to 1.45 ; $\mathrm{P}<0.0001)$. LVEDV was significantly less in CRP patients (SMD: $-0.31 ; 95 \% \mathrm{CI}:-0.59$ to -0.02 , fixed effect model $\mathrm{P}<0.05$ ). WMSI was significantly less in CRP patients (SMD: $-0.41 ; 95 \% \mathrm{CI}-0.78$ to -0.05 , $\mathrm{P}=0.024)$. CRP improves cardiac function in post $\mathrm{MI}$ patients. This may explain the reported improvement of functionality and mortality among those patients. Further randomized trials may help evaluate the longterm benefits of CRP.
\end{abstract}

Keywords: Cardiac rehabilitation program (CRP); post myocardial infarction; ejection fraction (EF\%)

Submitted Jul 01, 2019. Accepted for publication Aug 08, 2019.

doi: $10.21037 /$ atm.2019.08.64

View this article at: http://dx.doi.org/10.21037/atm.2019.08.64

(c) Annals of Translational Medicine. All rights reserved. 


\section{Introduction}

Exercise-based cardiac rehabilitation results in improved exercise capacity (1) and in reduction of mortality in patients after acute myocardial infarction (AMI) as seen in a recent Cochrane review (2-4). It has also been suggested to improve cardiovascular functional capacity, quality of life, risk factors modification and psychological profile (5).

Various factors have been suggested to explain the effect of cardiac rehabilitation on cardiac function. The extent of ventricular remodeling has been reported to be an important prognostic factor associated with cardiac function after AMI, more published studies have demonstrated that, in AMI patients with left ventricular dysfunction, exercise training not only does not contribute but may even prevent worsening of ventricular remodeling (6). Proponents of this observation have suggested that improvement in impaired sympatho-vagal balance might be the underlying mechanism. In addition, exercise training is thought to contribute to improvement in heart rate recovery (HRR), a powerful and easily measured index of vagal tone in AMI patients (7).

Early studies of a cardiac rehabilitation program (CRP) done nearly two decades ago in the form of exercise training and education classes have shown that these programs improve exercise capacity in patients with coronary heart disease (CHD) (8-10). However, these studies were limited by either small sample size $(8,9)$ or were uncontrolled $(10)$.

Large, randomized, controlled studies were needed to confirm the benefit of CRP on exercise capacity. Furthermore, the mechanisms of improvement of exercise tolerance after CRP are not fully understood. The objective improvement in cardiac function after exercise training is controversial (11-13). The aim of this study was to systematically review the available data in order to evaluate the effects of a supervised exercise program in improving cardiac functions for patients post AMI in terms of cardiac objective evaluations detected on echo as well as stress testing.

\section{Methods}

\section{Literature search and selection of studies}

Online search of MEDLINE, EMBASE, PubMed, Google Scholar, Scopus, and the Cochrane database was done, with identification of all English language published articles comparing the cardiac physiologic measurements in post AMI patients after treatment followed with or without CRP.
The search was conducted using the following MeSH terms "Cardiac Rehabilitation Program" AND "exercise based rehabilitation" AND "acute myocardial infarction", "cardiac function", "cardiac physiology". We used the relatedarticles function to expand the search from relevant studies. All publications were thoroughly reviewed. The search was last performed on 07/01/2017. Bibliographies of reviewed papers were additionally screened for any other studies that could be included.

\section{Outcomes of interest}

Only studies reporting on the comparison between AMI patients who had exercise based CRP and those who did not were included, regardless of mode of post AMI treatment [i.e., medical, surgical revascularization or percutaneous coronary intervention (PCI)]. The primary endpoint of the study was resting and maximum heart rate (HR), ejection fraction $(\mathrm{EF} \%)$, peak $\mathrm{VO}_{2}$, left ventricular end diastolic volume (LVEDV), and wall motion score index (WMSI) in cardiac rehabilitation patients versus control.

\section{Inclusion criteria}

In order to be included in the analysis, studies had to compare the outcome measures mentioned above between patients who underwent CRP post AMI and those who did not and report at minimum one of the outcomes of interest. If an institution reported two studies, we selected either the study of better quality, larger sample size or the most recent. We excluded studies if they were either non-comparative, published as case series, did not report on outcomes of interest for the two groups, or there was an overlap between patient cohorts, authors, or institutions.

\section{Definitions}

* CRP was defined as a training period that ranged between 1 to 4.5 months, between 10 minutes to 2 hours for 3 days a week to daily, mainly on a bicycle ergometer, or walking on tread mill, with targets of $60-70 \%$ of peak $\mathrm{VO}_{2}$ was used in some studies, others used standard training protocols (e.g., Heleniski);

* Exercise testing for obtaining results: bicycle ergometric test was performed starting by 1 minute warm up, then a work load ranging from 1 Workload (W) to $25 \mathrm{~W}$ increase every 1 to 2 minutes, to achieve a $60-70 \%$ peak $\mathrm{VO}_{2}$ uptake which was the limit in 


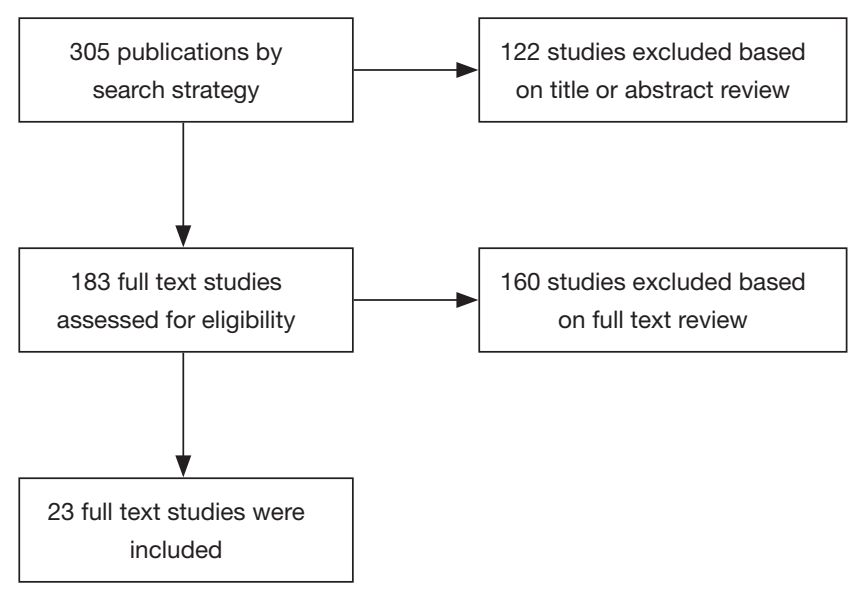

Figure 1 Systematic literature search and selection strategy.

some studies. The test ends by cooling down for 1 minute. In some studies, the process was supervised by cardiologist, and therapists;

* WMSI: wall motion score index was observed and the motion was graded as normal 0 , hyperkinesia 1 , akinesia 2, dyskinesia 3. The less the WMSI the better the wall motion. It was confirmed by nuclear test in some studies;

* LVEDV: this was calculated in some studies, the smaller the volume the better the effect of rehabilitation and the more the remodeling effect is reversed;

Peak $\mathrm{VO}_{2}$ : peak $\mathrm{O}_{2}$ uptake (how much $\mathrm{O}_{2}$ per minute), it expresses $\mathrm{Max}_{2}$ consumption even if the work load is increased, it provides assessment of functional capacity, it was used as a good short term predictor of mortality, calculated by averaging the final $20-30$ seconds in the exercise test.

\section{Data extraction}

Between two reviewers (I Kirolos and F Pendola), the studies were independently searched, and data was extracted from each study as follows: study characteristics (first author, study design and year of publication) and population characteristics (number of patients included, age, sex, and comorbidity characteristics). The outcomes of interest measured included resting and maximum $\mathrm{HR}$, peak $\mathrm{VO}_{2}$, EF\%, WMSI, LVEDV in cardiac rehabilitation patients versus control. We converted continuous data that was reported as median and range to the mean and standard deviation.

\section{Statistical analysis}

The software Stata 12 (StataCorp LP, College Station, TX, USA) was used for statistical analysis. For continuous outcomes, standardized mean difference (SMD) and 95\% confidence intervals (CI) were used to analyze data as the summary statistic. SMDs greater than zero suggested the degree to which treatment is more efficacious than placebo. Conversely, SMDs lower than zero suggested the degree to which treatment is less efficacious than placebo (14). All $\mathrm{P}$ values of $<0.05$ (two tailed) were considered statistically significant. For the meta-analysis, we used both the fixed and random-effects models of Mantel-Haenszel and DerSimonian-Laird $(15,16)$. When clinical or statistical heterogeneity was encountered, the random effects model was used. The $\chi^{2}$ (or Cochran Q statistic) and $\mathrm{I}^{2}$ statistic was used to quantify statistical heterogeneity across studies. The formula $[(\mathrm{Q}-\mathrm{df} / \mathrm{Q}) \times 100]$ was used to derive the $\mathrm{I}^{2}$ statistic from the $\mathrm{Q}$ statistic to provide a measure of the proportion of the overall variation that can be attributed to heterogeneity among the studies. A Q statistic that showed $\mathrm{P}<0.20$ suggested absence of homogeneity, and an $\mathrm{I}^{2}$ statistic exceeding 50\% suggested significant heterogeneity. Subgroup analyses were selectively done as needed. The 25 -item CONSORT checklist was used for quality assessment of the prospective studies (17).

\section{Results}

\section{Literature search}

Initial search yielded 305 studies. Only 183 studies fit our selection criteria based on their titles and abstracts. Full text of those was reviewed. Out of those, 160 studies were excluded according to inclusion and exclusion criteria. Therefore, 23 studies totaling 1,683 patients were selected to be included in the meta-analysis (Figure 1); 827 (49\%) patients were enrolled in a CRP while $855(51 \%) \mathrm{did}$ not receive the intervention. Median age of patients was 58 years with equal male to female distribution in both intervention and control groups. There was no difference between the two groups regarding age, sex, comorbidities, severity of CAD, baseline EF\% or HR (18-40) (Table 1).

\section{Outcomes of interest}

Resting HR: post exercise resting HR was measured and reported in 15 out of 23 studies included in our analysis. Meta-analysis of included studies' data showed that CRP 
Table 1 Characteristics of included studies

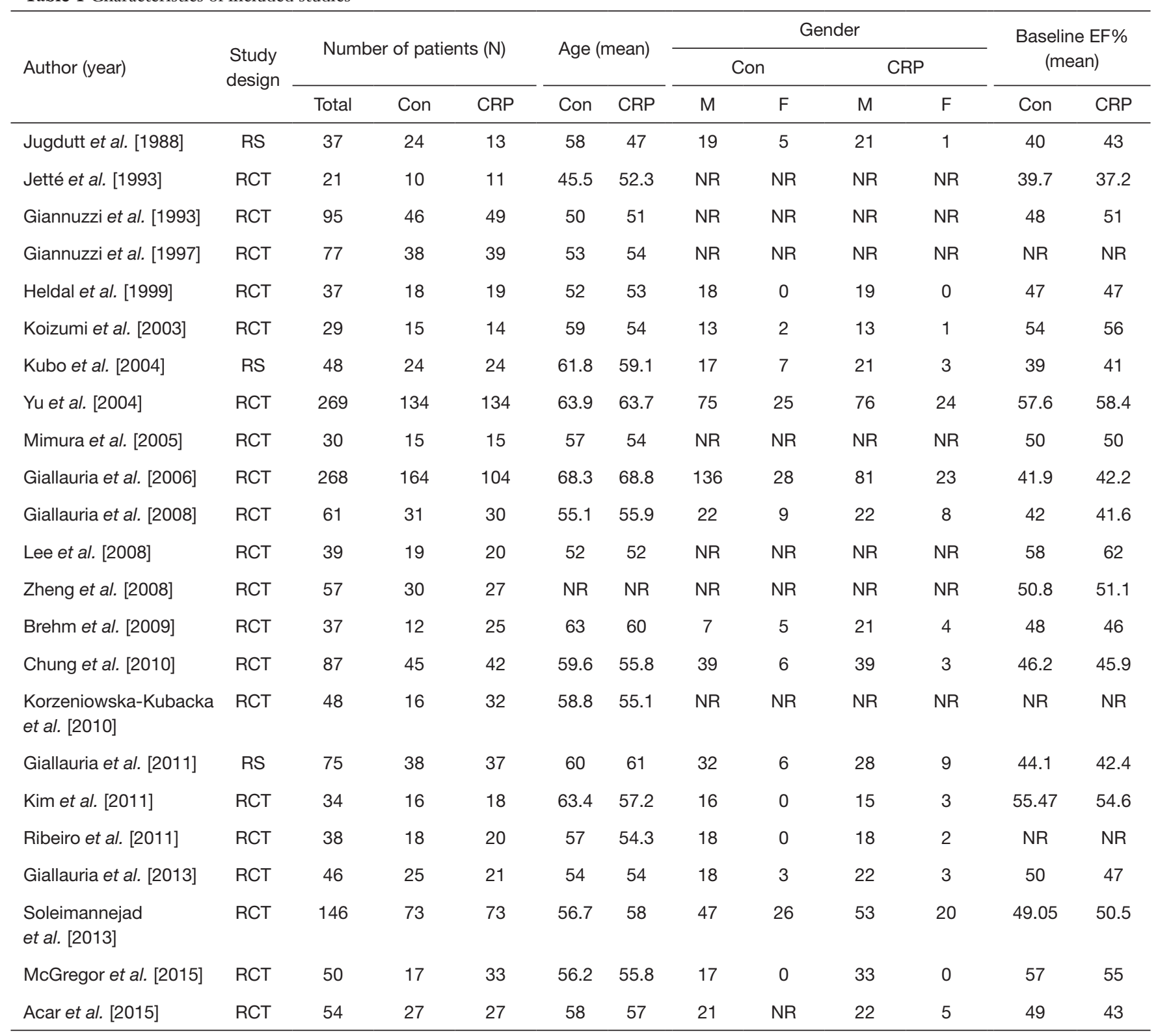

RS, retrospective study; RCT, randomized controlled trial; Con, control; NR, not recorded; CRP, cardiac rehabilitation program; EF\%, ejection fraction percentage.

patients had lower post intervention resting HR with a median of $69 \mathrm{bpm}$ (R: 56-76) compared to non-CRP patients where median was $71 \mathrm{bpm}$ (R: 54-79). Despite close numbers, the difference was statistically significant (SMD: -0.59 ; 95\% CI: -0.73 to -0.46 ; fixed effect model $\mathrm{P}<0.05$ ) (Figure 2). Of note, heterogeneity between studies was significant $\left(\mathrm{P}<0.001, \mathrm{I}^{2}=86.8 \%\right)$. Subgroup analysis of high-quality studies showed similar results.

EF\%: 17 of 23 studies reported on EF\% measurement after implementing CRP compared to control, this was measured in comparison to pre-program measurement which was not different in either groups. Meta-analysis data demonstrated that CRP trained patients had significantly higher post-stress EF\% compared to non-CRP patients (SMD: $0.21 ; 95 \%$ CI: 0.02 to $0.40, \mathrm{P}=0.032$ ). Subgroup analysis of patients who had $\mathrm{EF} \%>45 \%$ at baseline showed similar results (SMD: 0.24; 95\% CI: 0.01 to $0.48, \mathrm{P}=0.04$ ), while subgroup analysis of patients who had $\mathrm{EF} \%<45 \%$ 


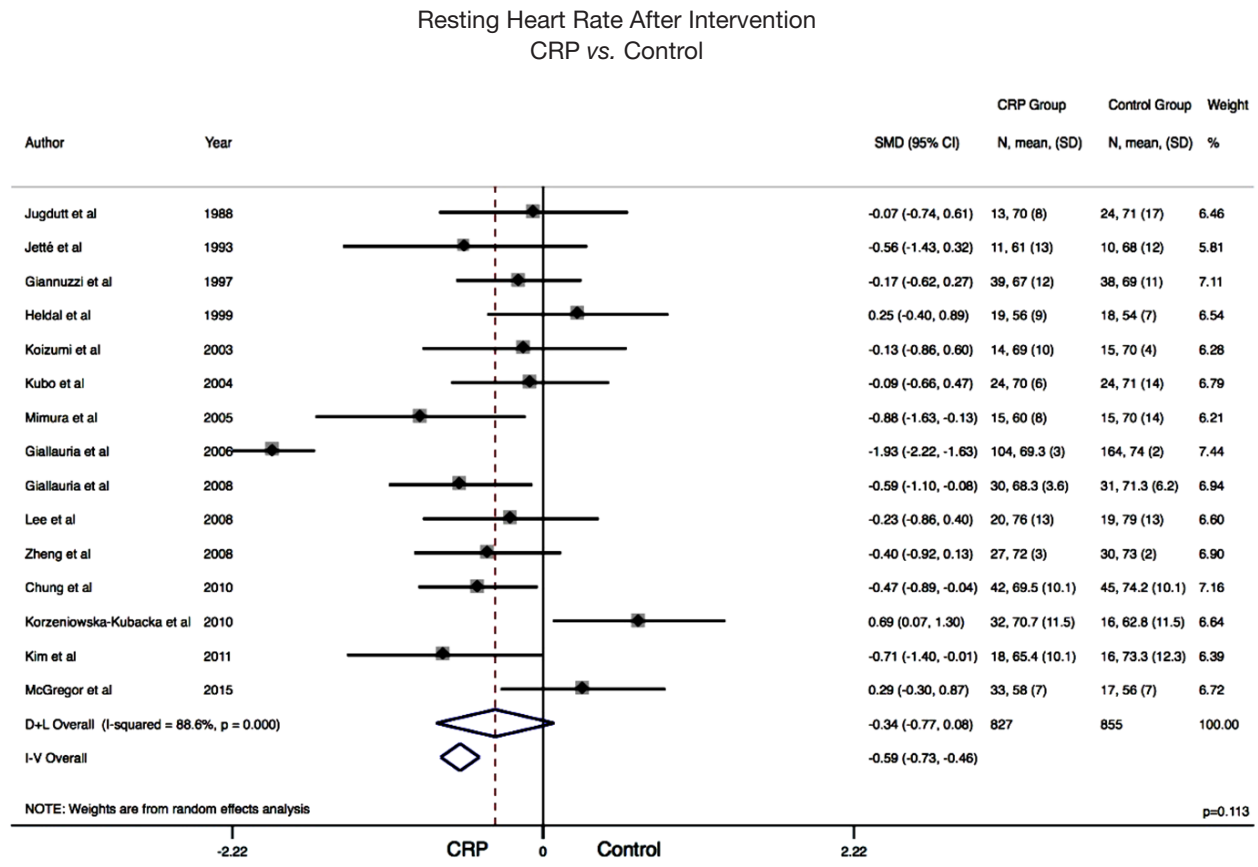

Figure 2 Forest plot showing significantly lower resting HR in CRP patient group (Fixed effects model). HR, heart rate; CRP, cardiac rehabilitation program; D+L, Der-Simonian and Laird (random effects model); I-V, inverse variance (fixed effects model).

at baseline showed no statistically significant difference between the groups (SMD: 0.17; CI: -0.04 to $0.37, \mathrm{P}=0.497$ ) (Figure 3). Heterogeneity was significant in the overall and baseline $\mathrm{EF} \%>45 \%\left(\mathrm{I}^{2}=67.7 \%, 71.4 \%\right.$, respectively, $\mathrm{P}<0.001$ ) while it was insignificant in the subgroup of patients with baseline $\mathrm{EF}<45 \%\left(\mathrm{I}^{2}=49 \%, \mathrm{P}=0.11\right)$.

Peak $\mathrm{VO}_{2}: 12$ of 23 studies included reported on Peak $\mathrm{VO}_{2}$. Meta-analysis of included data showed that it was significantly improved by CRP compared to control (SMD: 1.00 ; 95\% CI: 0.56 to $1.45, \mathrm{P}<0.0001$ ) (Figure 4). Heterogeneity was significant $\left(\mathrm{I}^{2}=87.6 \%, \mathrm{P}<0.001\right)$.

LVEDV: only 4 out of 23 studies reported on LVEDV. Meta-analysis of included data showed that LVEDV was significantly less in CRP patients compared to controls on fixed effect models (SMD: -0.31 ; $95 \%$ CI: -0.59 to -0.02 ; $\mathrm{P}<0.05$ ) (Figure 5). Heterogeneity was also significant $\left(\mathrm{I}^{2}=78.1 \%, \mathrm{P}=0.003\right)$.

WMSI: only 2 of 23 studies included in this analysis reported on WMSI. Meta-analysis of included data showed that WMSI was significantly less in CRP patients compared to controls (SMD: $-0.41 ; 95 \% \mathrm{CI}:-0.78$ to $-0.05, \mathrm{P}=0.024$ ) (Figure 6). Heterogeneity was not significant $\left(\mathrm{I}^{2}=0 \%\right.$, $\mathrm{P}=0.967)$.

The quality of studies in the overall analysis was assessed by CONSORT checklist for RCTs. This showed that most RCTs were of relatively good quality with a mean score of 20 and above. Subgroup analysis was done for higher quality studies. This showed the same trend observed in the overall analysis with achievement of statistical significance in most outcomes. This denotes validity of the overall analysis and rules out the possibility of being skewed by studies of lesser quality.

\section{Discussion}

Our results showed that CRP in post AMI patients significantly improved all surrogate markers of improved cardiac physiologic functions, including lower post exercise resting HR, increased their EF\%, lower WMSI and LVEDV, and significantly improved peak $\mathrm{VO}_{2}$.

Study data reviewed from various clinical trials have demonstrated significant reductions in cardiovascular as well as all-cause mortality in patients with coronary artery disease who have been part of an exercise-based CR programs $(4,5)$. In terms of affecting the post exercise resting HR, Mimura et al. (26) suggested that exercise training decreases directly measured central sympathetic nerve outflow in patients with AMI. The sympatho-inhibitory effect of exercise training 
Post-Stress Ejection Fraction

CRP vs. Control

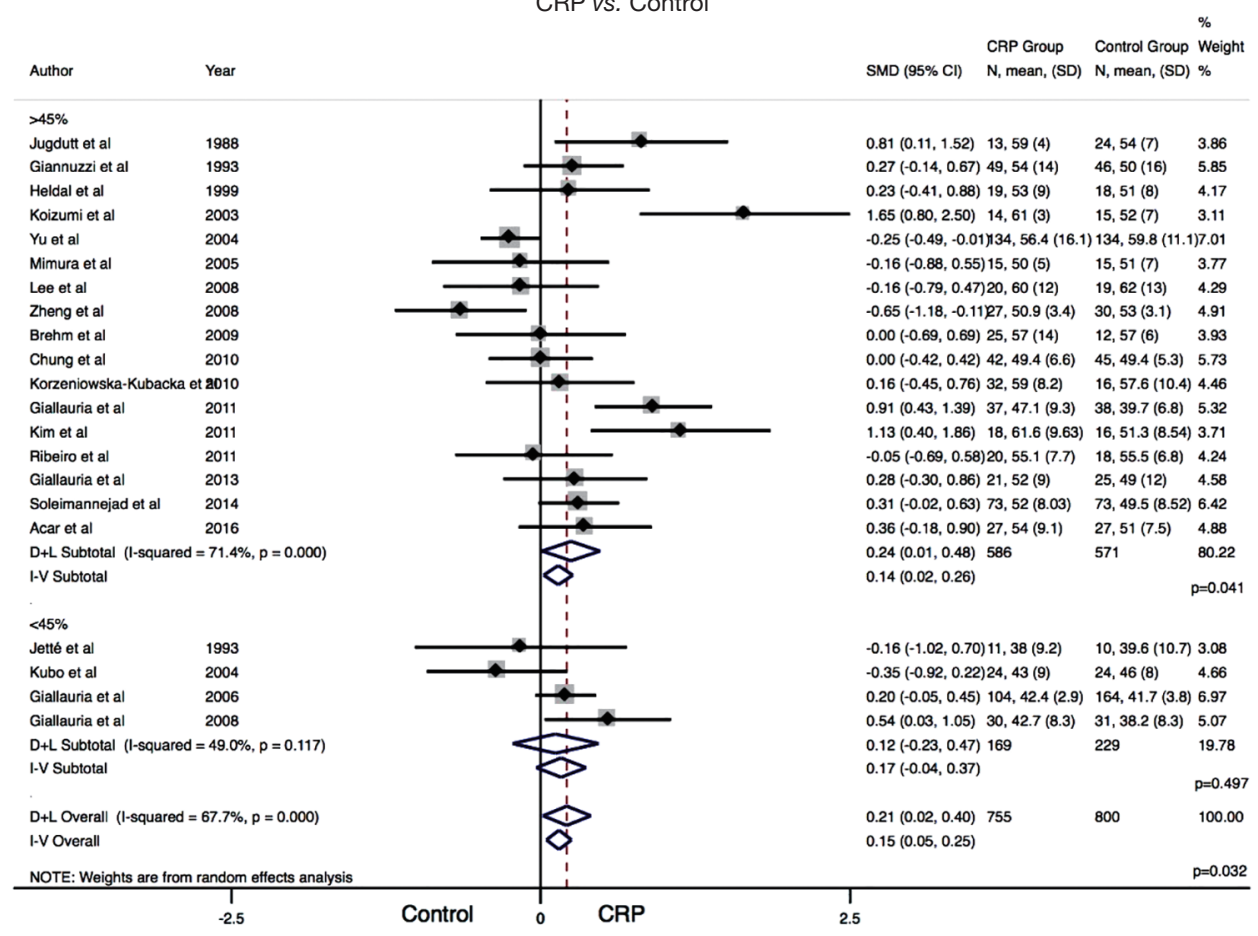

Figure 3 Forest plot showing significantly higher EF\% in CRP patient group compare to controls, difference was not significant in patients with EF less than 45\%. EF\%, ejection fraction percentage; CRP, cardiac rehabilitation program; D+L, Der-Simonian and Laird (random effects model); I-V, inverse variance (fixed effects model).

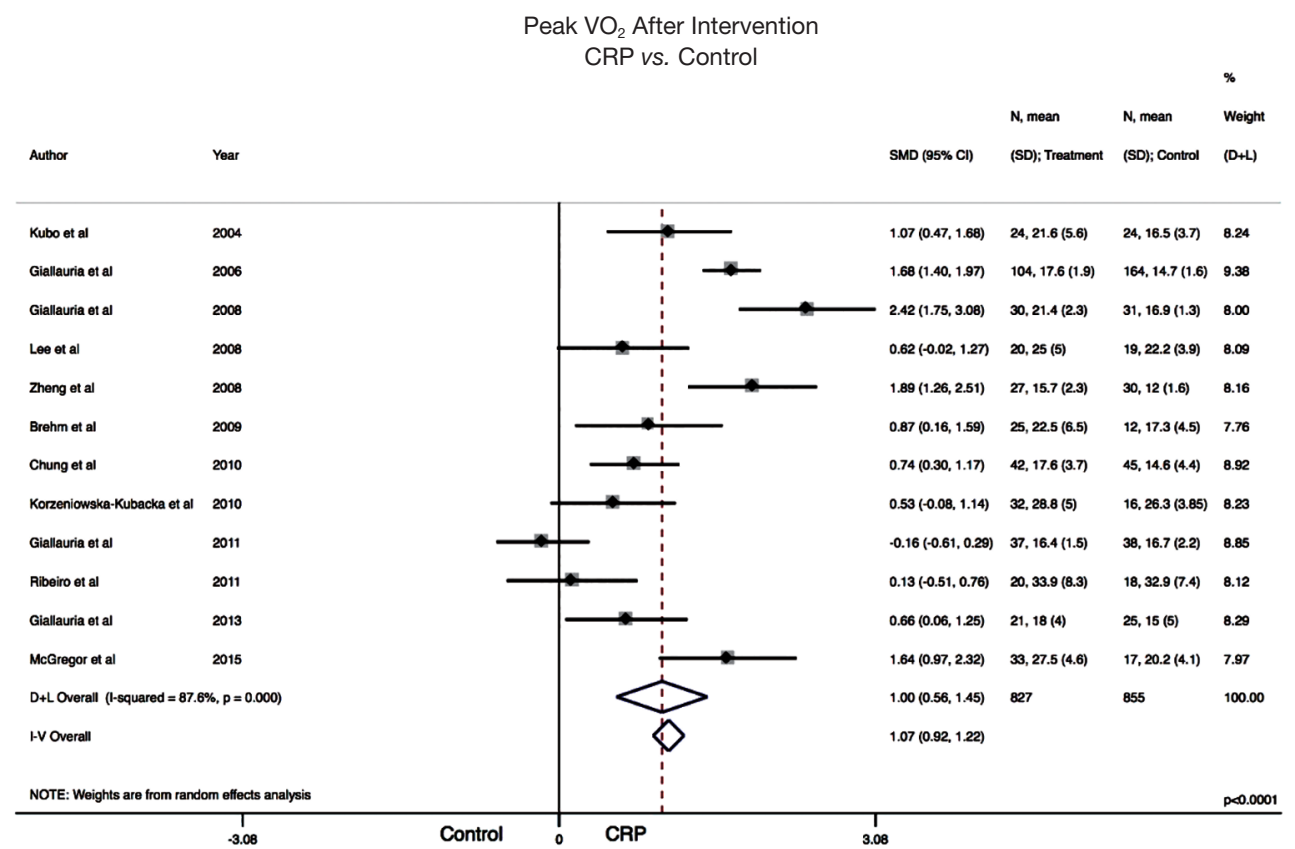

Figure 4 Forest plot showing significantly higher Peak $\mathrm{VO}_{2}$ in $\mathrm{CRP}$ patient group; $\mathrm{VO}_{2}$, maximal oxygen consumption, maximal oxygen uptake; CRP, cardiac rehabilitation program; D+L, Der-Simonian and Laird (random effects model); I-V, inverse variance (fixed effects model). 


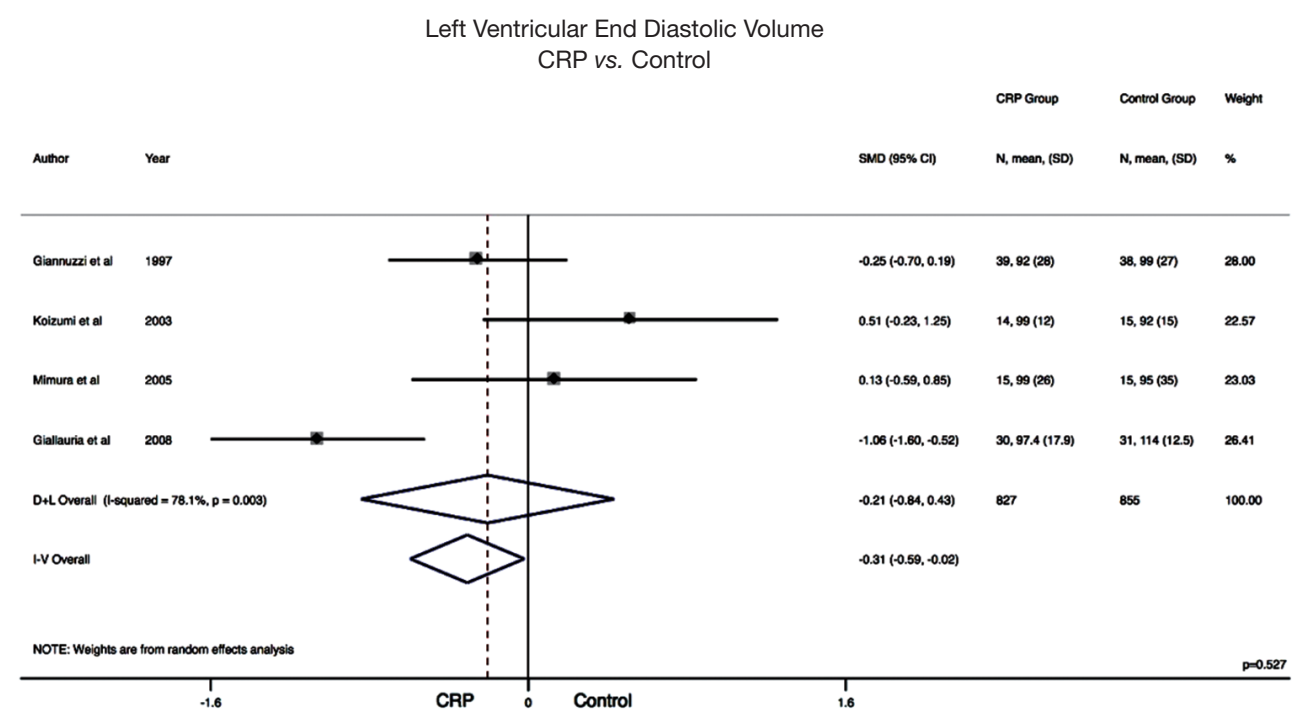

Figure 5 Forest plot showing significantly lower LVEDV in CRP patient group (fixed effects model). LVEDV, left ventricular end diastolic volume; CRP, cardiac rehabilitation program; D+L: Der-Simonian and Laird (random effects model); I-V, inverse variance (fixed effects model).

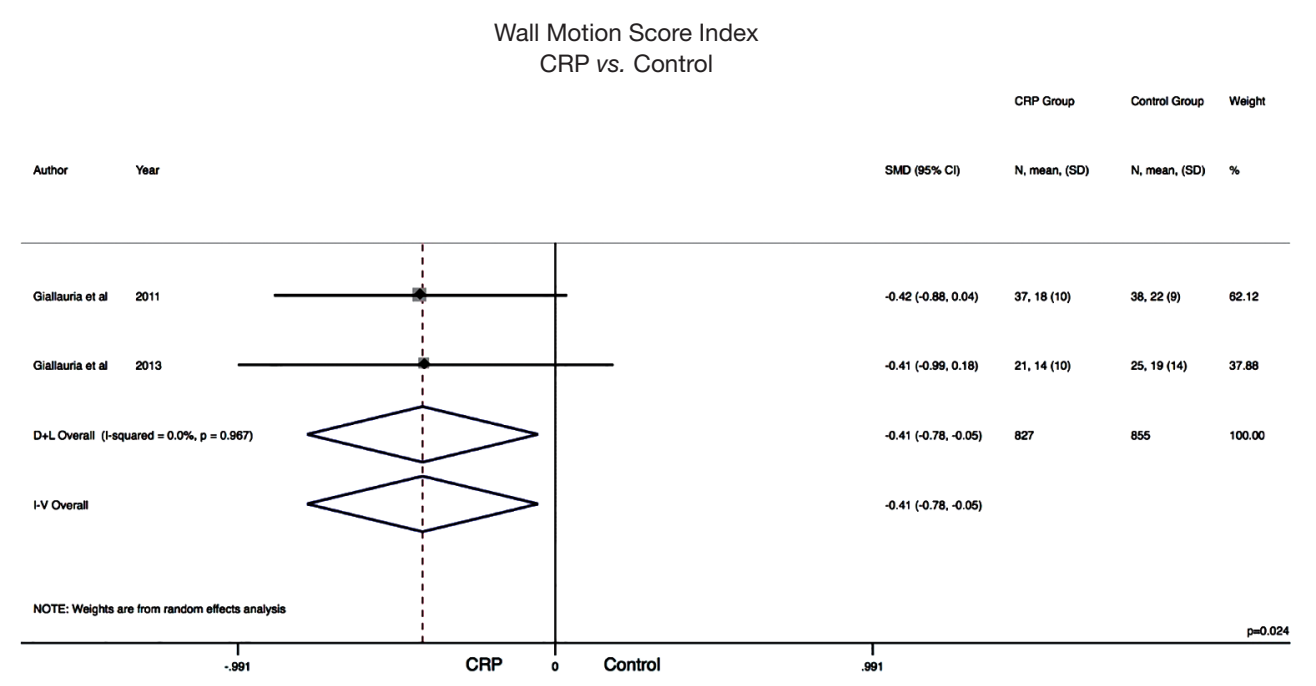

Figure 6 Forest plot showing significantly lower WMSI in CRP patient group. WMSI, Mall Motion Score Index; CRP, cardiac rehabilitation program; D+L, Der-Simonian and Laird (random effects model); I-V, inverse variance (fixed effects model).

may contribute to the known beneficial effects of exercise training on survival in patients with uncomplicated AMI, at least in part. On the other hand, Giallauria et al. (41) also argued that despite the repeated sympathetic activity and increased adrenalin levels during exercise that may result in stimulation of the cytotoxic effect and the apoptosis process in the myocardial cell, the eventual ability of exercise to indirectly stimulate vagal nerve activity leads to a relative bradycardia by directly (on one side) acting on the A-V node and indirectly (on the other side) by inhibiting back the sympathetic activity, which in turn will decrease the cardiac work load. In addition, Ribeiro et al. (36) suggested that baroreceptors stimulation by exercise, increases the vagal tone which decreases the $H R$, and secondly that one of the harmful effects of elevated HR is the instability of atherosclerotic plaques, which is subsequently avoided by 
a slower resting HR. The results of our analysis resonate with these suggested mechanisms where on pooling data from 15 out of 23 studies included, resting HR post exercise was shown to be significantly less in CRP patients compared to controls. In spite that this result supports the benefit of CRP, however this result should be viewed with caution as there was significant heterogeneity between the studies reporting on this outcome. In addition, its clinical significance is uncertain at the values detected.

In the current analysis, 17 out of 23 studies reported on $\mathrm{EF} \%$ which was found to be significantly improved in cardiac rehab patients compared to control group post exercise, of note these results are rather more significant in those patients who had a baseline $\mathrm{EF}>45 \%$. This may suggest a bigger benefit of CRP in this subgroup of patients compared to those with $\mathrm{EF}<45 \%$ post MI. Koizumi et al. and also Zheng et al. have suggested this may be due to improvement of oxidative metabolism or other neuro-humoral factors in the otherwise non-infarcted myocardium. This includes a central effect, i.e., the salvaged myocardium that escaped from ischemia, as well as a peripheral adaptation effect $(23,30)$.

As for peak $\mathrm{VO}_{2}, 12$ of our 23 included articles reported on it where the pooling of data showed statistically significant increase in peak $\mathrm{VO}_{2}$ in CRP patients. This is in line with literature on chronic heart failure patients whereas Jetté et al. (19) reported that there is an improvement in exercise performance capacity in CRP patients when compared to control group. It is well known that improved capillary supply and increased oxidative enzymes are important factors of dynamic exercise performance $(42,43)$. The improvement in peak $\mathrm{VO}_{2}$ in CRP patients may be attributed to an increase in peak blood flow to active skeletal muscles and a more efficient peripheral oxygen extraction, leading to reduced myocardial oxygen demand. Moreover, Giallauria et al. (27) studied functional capacity is a well-known player in cardiovascular risk factors in older patients, they concluded that functional capacity is best measured by the peak $\mathrm{VO}_{2}$, and that exercise was seen to increase peak $\mathrm{VO}_{2}$, then exercise will increase cardiac functional capacity and decrease cardiovascular risks, particularly in older patients.

Only 4 out of the 23 articles included in this analysis reported on LVEDV, which was significantly less in CRP patients, 4 other studies reported on LVEDD (diameter) showing similar trend of results to our analysis of LVEDV, yet we did not include those in the same figures as they used diameter length as opposed to ventricular volume units.
Chung et al. (32) suggested that the remodeling process that occurs after AMI affects the diastolic filling capacity by decreasing it, which in turn is a predictor of myocardial cell death, and eventually heart failure. Moreover, Yu et al. (25) reported on "Abnormal Relaxation Pattern" which only increased in control group, however in CRP group the decline in cardiac filling (diastolic dysfunction) in other words the increase in "Abnormal Relaxation Pattern", was not seen. Other authors suggested there was an element of "Revered LV Remodeling" caused the by CRP program. This was achieved through neurohormone factor, "improved endothelial function", and "autonomic adjustment". It also can be measured through the fall in pro-brain natriuretic peptide (pro-bnp) and the decrease in LVEDV $(28,30)$.

WMSI was reported in only 2 of our included studies, score was lower in CRP patients denoting improved wall motion, a few other studies reported on $\%$ wall motion abnormality and seem to have the same trend of the results of our analysis of WMSI; Giallauria et al. (41) studying the factors contributing to that and suggested that exercise increases myocardial perfusion which in turn increases the $\mathrm{LV}$ myocardial wall motion and thus decrease the score index. This is associated with decreased unfavorable LV remodeling and with the improvement of cardiovascular functional capacity.

Limitations of this study include the relatively small sample size of included studies, with some minor differences between the protocols followed in the CRP program and its length. Not all studies reported on all outcomes of interest taking away from the power of the analysis. In addition, there was significant heterogeneity between the studies in different outcomes, therefore results should not be over interpreted.

\section{Conclusions}

CRP objectively improves various aspects of functional cardiac capacity in post MI patients, this is mediated through higher EF and peak $\mathrm{VO}_{2}$, lower resting HR, LVEDV and WMSI. This effect may explain the reported improvement of functionality and mortality among those patients. Further randomized trials may help evaluate the long-term benefits of CRP.

\section{Acknowledgments}

None. 


\section{Footnote}

Conflicts of Interest: The authors have no conflicts of interest to declare.

Ethical Statement: The authors are accountable for all aspects of the work in ensuring that questions related to the accuracy or integrity of any part of the work are appropriately investigated and resolved.

\section{References}

1. Ades PA, Green NM, Coello CE. Effects of exercise and cardiac rehabilitation on cardiovascular outcomes. Cardiol Clin 2003;21:435-48.

2. O'Connor GT, Buring JE, Yusuf S, et al. An overview of randomized trials of rehabilitation with exercise after myocardial infarction. Circulation 1989;80:234-44.

3. Oldridge NB, Guyatt GH, Fischer ME, et al. Cardiac rehabilitation after myocardial infarction. Combined experience of randomized clinical trials. JAMA 1988;260:945-50.

4. Heran BS, Chen JM, Ebrahim S, et al. Exercise-based cardiac rehabilitation for coronary heart disease. Cochrane Database Syst Rev 2011;7:CD001800.

5. Taylor RS, Brown A, Ebrahim S, et al. Exercise-based rehabilitation for patients with coronary heart disease: systematic review and meta-analysis of randomized controlled trials. Am J Med 2004;116:682-92.

6. van Tol BA, Huijsmans RJ, Kroon DW, et al. Effects of exercise training on cardiac performance, exercise capacity and quality of life in patients with heart failure: a metaanalysis. Eur J Heart Fail 2006;8:841-50.

7. Sato S, Makita S, Majima M. Additional physical activity during cardiac rehabilitation leads to an improved heart rate recovery in male patients after coronary artery bypass grafting. Circ J 2005;69:69-71.

8. Ståhle A, Mattsson E, Ryden L, et al. Improved physical fitness and quality of life following training of elderly patients after acute coronary events: a 1 year follow-up randomized controlled study. Eur Heart J 1999;20:1475-84.

9. Hofman-Bang C, Lisspers J, Nordlander R, et al. Two-year results of a controlled study of residential rehabilitation for patients treated with percutaneous transluminal coronary angioplasty: a randomized study of a multifactorial programme. Eur Heart J 1999;20:1465-74.

10. Maines TY, Lavie CJ, Milani RV, et al. Effects of cardiac rehabilitation and exercise programs on exercise capacity, coronary risk factors, behavior, and quality of life in patients with coronary artery disease. South Med J 1997;90:43-9.

11. Jensen D, Atwood JE, Froelicher V, et al. Improvement in ventricular function during exercise studied with radionuclide ventriculography after cardiac rehabilitation. Am J Cardiol 1980;46:770-7.

12. Cobb FR, Williams RS, McEwan P, et al. Effects of exercise training on ventricular function in patients with recent myocardial infarction. Circulation 1982;66:100-8.

13. Verani MS, Hartung GH, Hoepfel-Harris J, et al. Effects of exercise training on left ventricular performance and myocardial perfusion in patients with coronary artery disease. Am J Cardiol 1981;47:797-803.

14. Faraone SV. Interpreting estimates of treatment effects: implications for managed care. P T 2008;33:700-11.

15. Mantel N, Haenszel W. Statistical aspects of the analysis of data from retrospective studies of disease. J Natl Cancer Inst 1959;22:719-48.

16. DerSimonian R, Laird N. Meta-analysis in clinical trials. Control Clin Trials 1986;7:177-88.

17. Moher D, Hopewell S, Schulz KF, et al. CONSORT 2010 explanation and elaboration: Updated guidelines for reporting parallel group randomised trials. BMJ 2010;340:c869.

18. Jugdutt BI, Michorowski BL, Kappagoda CT. Exercise training after anterior $\mathrm{Q}$ wave myocardial infarction: importance of regional left ventricular function and topography. J Am Coll Cardiol 1988;12:362-72.

19. Jetté M, Heller R, Landry F, et al. Randomized 4-week exercise program in patients with impaired left ventricular function. Circulation 1991;84:1561-7.

20. Giannuzzi P, Tavazzi L, Temporelli PL, et al. Long-term physical training and left ventricular remodeling after anterior myocardial infarction: results of the Exercise in Anterior Myocardial Infarction (EAMI) trial. EAMI Study Group. J Am Coll Cardiol 1993;22:1821-9.

21. Giannuzzi P, Temporelli PL, Corrà U, et al. Attenuation of unfavorable remodeling by exercise training in postinfarction patients with left ventricular dysfunction: results of the Exercise in Left Ventricular Dysfunction (ELVD) trial. Circulation 1997;96:1790-7.

22. Heldal M, Rootwelt K, Sire S, et al. Short-term physical training reduces left ventricular dilatation during exercise soon after myocardial infarction. Scand Cardiovasc J 2000;34:254-60.

23. Koizumi T, Miyazaki A, Komiyama N, et al. Improvement of left ventricular dysfunction during exercise by walking 
in patients with successful percutaneous coronary intervention for acute myocardial infarction. Circ J 2003;67:233-7.

24. Kubo N, Ohmura N, Nakada I, et al. Exercise at ventilatory threshold aggravates left ventricular remodeling in patients with extensive anterior acute myocardial infarction. Am Heart J 2004;147:113-20.

25. Yu CM, Li LS, Lam MF, et al. Effect of a cardiac rehabilitation program on left ventricular diastolic function and its relationship to exercise capacity in patients with coronary heart disease: experience from a randomized, controlled study. Am Heart J 2004;147:e24.

26. Mimura J, Yuasa F, Yuyama R, et al. The effect of residential exercise training on baroreflex control of heart rate and sympathetic nerve activity in patients with acute myocardial infarction. Chest 2005;127:1108-15.

27. Giallauria F, Lucci R, Pietrosante M, et al. Exercisebased cardiac rehabilitation improves heart rate recovery in elderly patients after acute myocardial infarction. J Gerontol A Biol Sci Med Sci 2006;61:713-7.

28. Giallauria F, Cirillo P, Lucci R, et al. Left ventricular remodeling in patients with moderate systolic dysfunction after myocardial infarction: favourable effects of exercise training and predictive role of $\mathrm{N}$-terminal pro-brain natriuretic peptide. Eur J Cardiovasc Prev Rehabil 2008;15:113-8.

29. Lee BC, Chen SY, Hsu HC, et al. Effect of cardiac rehabilitation on myocardial perfusion reserve in postinfarction patients. Am J Cardiol 2008;101:1395-402.

30. Zheng H, Luo M, Shen Y, et al. Effects of 6 months exercise training on ventricular remodeling and autonomic tone in patients with acute myocardial infarction and percutaneous coronary intervention. J Rehabil Med 2008;40:776-9.

31. Brehm M, Picard F, Ebner P, et al. Effects of exercise training on mobilization and functional activity of bloodderived progenitor cells in patients with acute myocardial infarction. Eur J Med Res 2009;14:393-405.

32. Chung CC, Huang WC, Chiou KR, et al. Ratio of early mitral inflow peak velocity to flow propagation velocity predicts training effects of cardiac rehabilitation in patients after acute myocardial infarction. J Rehabil Med 2010;42:232-8.

33. Korzeniowska-Kubacka I, Bilińska M, Michalak E, et al. Influence of exercise training on left ventricular diastolic function and its relationship to exercise capacity in patients after myocardial infarction. Cardiol J 2010;17:136-42.

34. Giallauria F, Cirillo P, D'agostino M, et al. Effects of exercise training on high-mobility group box-1 levels after acute myocardial infarction. J Card Fail 2011;17:108-14.

35. Kim C, Kim DY, Lee DW. The impact of early regular cardiac rehabilitation program on myocardial function after acute myocardial infarction. Ann Rehabil Med 2011;35:535-40.

36. Ribeiro F, Alves AJ, Teixeira M, et al. Exercise training enhances autonomic function after acute myocardial infarction: a randomized controlled study. Rev Port Cardiol 2012;31:135-41.

37. Giallauria F, Acampa W, Ricci F, et al. Exercise training early after acute myocardial infarction reduces stressinduced hypoperfusion and improves left ventricular function. Eur J Nucl Med Mol Imaging 2013;40:315-24.

38. Soleimannejad K, Nouzari Y, Ahsani A, et al. Evaluation of the effect of cardiac rehabilitation on left ventricular diastolic and systolic function and cardiac chamber size in patients undergoing percutaneous coronary intervention. J Tehran Heart Cent 2014;9:54-8.

39. Acar RD, Bulut M, Ergün S, et al. Evaluation of the effect of cardiac rehabilitation on left atrial and left ventricular function and its relationship with changes in arterial stiffness in patients with acute myocardial infarction. Echocardiography 2015;32:443-7.

40. McGREGOR G, Gaze D, Oxborough D, et al. Reverse left ventricular remodeling: effect of cardiac rehabilitation exercise training in myocardial infarction patients with preserved ejection fraction. Eur J Phys Rehabil Med 2016;52:370-8.

41. Giallauria F, Acampa W, Ricci F, et al. Effects of exercise training started within 2 weeks after acute myocardial infarction on myocardial perfusion and left ventricular function: a gated SPECT imaging study. Eur J Prev Cardiol 2012;19:1410-9.

42. Wilson JR, Martin JL, Schwartz D, et al. Exercise intolerance in patients with chronic heart failure: Role of impaired nutritive flow to skeletal muscle. Circulation 1984;69:1079-87.

43. Plyley MJ. Fine-tuning muscle capillary supply for maximum exercise performance. Perspect Cardiol 1990;6:25-34.

Cite this article as: Kirolos I, Yakoub D, Pendola F, Picado O, Kirolos A, Levine YC, Jha S, Kabra R, Cave B, Khouzam RN. Cardiac physiology in post myocardial infarction patients: the effect of cardiac rehabilitation programs - a systematic review and update meta-analysis. Ann Transl Med 2019;7(17):416. doi: 10.21037/atm.2019.08.64 